\section{Doris von Muralt Portmann}

\title{
Parästhesien bei Diabetes mellitus
}

\author{
Therapieansätze mit PADMA 28*
}

systemischen Low-level-Entzündungszustand (Abb. 1). Über längere Zeit führen diese Prozesse zu endothelialer Dysfunktion, Durchblutungsstörungen sowie Nerven- und Zellschädigung. Sie stellen damit die Ursache von diabetischen Makro- und Mikroangiopathien dar, zu denen z.B. periphere arterielle Verschlusskrankheit (PAVK), diabetische Retino-, Neuround Nephropathie sowie das diabetische Fusssyndrom gehören. doppelt so häufig wie bei Nichtdiabetikern), Hyperlipidämie und Adipositas sowie eine ganze Reihe von diabetischen Folgeerkrankungen breiten sich aufgrund eines veränderten Lebensstils immer weiter aus und erfordern in der Praxis ein ganzheitliches Diabetes-Management.

\section{Diabetische Folgeerkrankungen - eine gemeinsame Ursache?}

Hyperglykämie hat sowohl direkte als auch indirekte pathologische Prozesse zur Folge. Hier spielt die endogene, nichtenzymatische Glykation von Proteinen eine zentrale Rolle, die zur Bildung von «advanced glycation end products» (AGEs) führt. AGE-Bildung kommt auch bei Gesunden vor; bei Diabetikern wird sie aber durch oxidativen Stress und entzündliche Prozesse verstärkt und führt über Feedbackmechanismen zu einem proinflammatorischen Kreislauf [2]. Selbst bei gut eingestellten Patienten kommt es so zu einem chronischen,

*PADMED CIRCOSAN ${ }^{\circledR}$ steht speziell dem Arzt zur Verfügung. Es ist kassenzulässig und identisch mit PADMA ${ }^{\oplus} 28$.

\section{Parästhesien und Schmerzen in den Extremitäten}

Unangenehmes Kribbeln, Taubheitsgefühle, stechende oder dumpfe Schmerzen an Beinen und Füssen, manchmal auch an Armen und Händen, kommen bei Diabetikern häufig vor und können die Lebensqualität stark vermindern. Die Symptome wer-
Abb. 1. Pathogenetische Prozesse bei der Entstehung von diabetischen Folgeerkrankungen. Verschiedene Faktoren (weiss unterlegt) tragen $\mathrm{zu}$ Parästhesien und Schmerzen in den Extremitäten bei. den meist durch eine periphere diabetische Neuropathie verursacht, können jedoch auch durch Durchblutungsstörungen ausgelöst oder verstärkt werden, insbesondere beim Vorliegen einer PAVK (Abb. 1). Während durch arterielle Mangeldurchblutung ausgelöste Schmerzen bei Belastung auftreten, verstärken sich Symptome der peripheren Neuropathie im Allgemeinen in Ruhe, besonders nachts, was in schweren Fällen zu Schlafstörungen und Depression führen kann.

Ein weiterer Punkt, den es bei Parästhesien und verminderter Schmerzempfindung zu beachten gilt, ist die Gefahr, dass Läsionen nicht oder spät entdeckt werden. In Kombination mit der erhöhten Infektanfälligkeit und suboptimaler Gewebedurchblutung beim Diabetiker kann es zu Ulzera und zur Entwicklung eines diabetischen Fusssyndroms kommen.

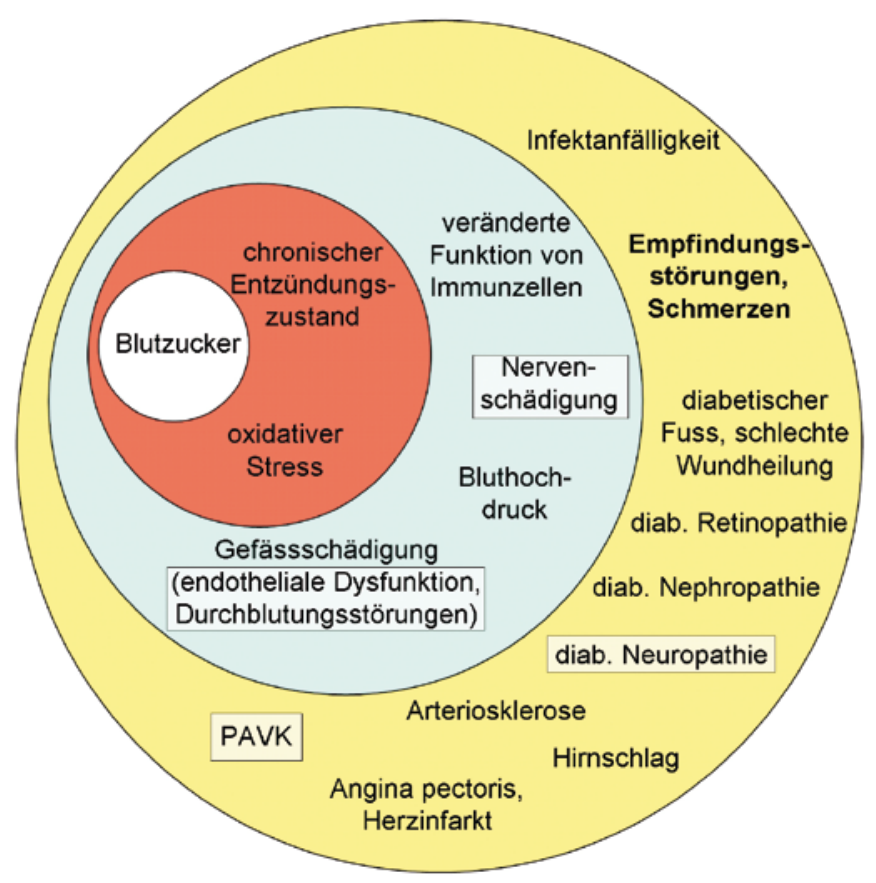

Dr. med. Doris von Muralt Portmann

Allgemeinmedizin FMH

Richigenstrasse 4, 3076 Worb, Schweiz

Tel. +41318392503 


\section{Ganzheitliches Diabetes- Management}

In der Behandlung des Diabetes steht die Kontrolle der Glykämie im Zentrum. In einem ersten Schritt wird versucht, diese durch eine Optimierung des Lebensstils (Ernährung, körperliche Aktivität, Reduzierung von Übergewicht, Nichtrauchen) zu erreichen. Die empfohlene Einstellung liegt gemäss Schweizerischer Diabetes-Gesellschaft im Allgemeinen bei einem HbAlc-Wert von <7\% [3]. Kann dieses Ziel nicht erreicht werden, stehen verschiedene antidiabetische Medikamente zur Senkung des Blutzuckers zur Verfügung.

Zusätzlich muss diabetischen Folgeerkrankungen adäquat vorgebeugt bzw. diese behandelt werden. Unter Berücksichtigung der komplexen $\mathrm{Pa}$ thogenese gibt es vielversprechende Präventions- und Behandlungsansätze aus der Phytotherapie. Sekundäre Pflanzenstoffe, wie z.B. Flavonoide, Tannine, Bitterstoffe und ätherische Öle, können den oxidativen Stress im Körper reduzieren und körpereigene antientzündliche Mechanismen stimulieren. Für verschiedene Pflanzenstoffe konnte zudem eine Hemmung der AGE-Bildung, ein pathogenetischer Schlüsselprozess bei der Entstehung von diabetischen Folgeerkrankungen, nachgewiesen werden [4].

\section{Ein Therapieansatz aus der Phytotherapie: PADMA 28}

Zur Behandlung von Diabetes-assoziierten Symptomen, wie z.B. Parästhesien, Kribbeln und Ameisenlaufen, bietet sich unter den phytotherapeutischen Arzneimitteln die Rezeptur PADMA 28 an. Neben seinen antioxidativen und antientzündlichen Eigenschaften konnte zudem ein Effekt bei Durchblutungsstörungen und PAVK gezeigt werden $[5,6]$, was bei der häufigen Komorbidität mit Arteriosklerose günstig ist. Im Folgenden werden zwei Patientenfälle aus der Praxis beschrieben, bei denen Diabetes-assozi- ierte Parästhesien und Schmerzen in den Extremitäten zusätzlich zur bestehenden Therapie mit PADMA 28 behandelt wurden.

\section{Fallberichte}

- Patient 1, 68 Jahre, BMI $25 \mathrm{~kg} / \mathrm{m}^{2}$, litt seit 13 Jahren an Diabetes mellitus Typ 2 und ausserdem an PAVK Fontaine Stadium IIb. Er wurde mit Insulin Mixtard und Insulin Actrapid behandelt und erhielt zur Plättchenaggregationshemmung prophylaktisch Azetylsalizylsäure $100 \mathrm{mg} /$ Tag. Wegen Gefühlsstörungen in den Füssen und Schmerzen in den Beinen wurde ihm zusätzlich PADMA $283 \times 2$ Tabletten/Tag verschrieben. Bei der Visite nach 6 Wochen waren die Beschwerden von mittelstarker auf schwache Intensität zurückgegangen. Bei der Kontrolle nach weiteren 3,5 Monaten war der Patient beschwerdefrei und die Dosierung wurde auf $3 \times 1$ Tablette/Tag reduziert (Tab. 1). Im Beobachtungszeitraum von weiteren 8 Monaten traten die Beschwerden unter dieser Erhaltungsdosis nicht wieder auf und die Behandlung mit PADMA 28 wurde zur Prävention weitergeführt.

- Patient 2, 57 Jahre, BMI 37,8 kg/m², litt seit 8 Jahren an Diabetes mellitus Typ 2, der mit Metformin $1000 \mathrm{mg} / \mathrm{Tag}$ und Pioglitazon $30 \mathrm{mg} /$ Tag behandelt wurde. Wegen mittelstarker Parästhesien in den Füssen und Dysästhesien in den Händen wurde ihm PADMA $283 \times 2$ Tabletten/Tag verschrieben. Nach 6 Wochen Behandlung hatten sich die Beschwerden deutlich reduziert und die Dosis wurde auf $2 \times 1$ Tablette/Tag herabgesetzt. Nach weiteren 6 Wochen war der Patient beschwerdefrei (Tab. 1). Die Behandlung mit PADMA 28 wurde in der Erhaltungsdosis von $2 \times 1$ Tablette/Tag fortgeführt.

Bei beiden Patienten zeigte sich bei der Visite nach 6 Wochen eine deutliche Verminderung der Parästhesien und der Schmerzen in den Beinen. In der weiteren Behandlung mit PADMA 28 verschwanden diese Beschwerden gänzlich. Da mit Diabetes mellitus die zugrunde liegende Pathologie weiter besteht, wurde nach Abklingen der Symptome die Behandlung mit PADMA 28 in der Erhaltungsdosis $3 \times 1$ bzw. $2 \times 1$ Tablette/Tag bis auf Weiteres fortgeführt. Unter dieser Dosierung sind die Beschwerden im Beobachtungszeitraum nicht wieder aufgetreten. Im ersten Fall, bei dem der Patient zusätzlich unter PAVK litt, erstreckte sich dieser über 13 Monate, was als gutes Resultat zu werten ist.

\section{Fazit für die Praxis}

Die Prävalenz von Diabetes mellitus Typ 2 nimmt zu und Experten sprechen bereits von einer «Diabetes-Epidemie». Der steigende Anteil an komorbiden Patienten und die Zunahme diabetischer Folgeerkrankungen machen für den Allgemeinpraktiker eine ganzheitliche Herangehensweise erforderlich. Neben der Anpassung von Lebensstilfaktoren können auch phytotherapeutische Arzneimittel im Diabetes-Management eingesetzt werden, die antioxidative und antientzündliche Eigenschaften haben und damit an den zentralen pathogenetischen Prozessen ansetzen. Das Beispiel des Einsatzes von PADMA 28 bei Diabetesassoziierten Parästhesien und Schmerzen zeigt, dass Phytotherapeutika ein vielversprechendes Potenzial in der Prävention und Behandlung von diabetischen Folgeerkrankungen haben.

\section{Literatur}

1 Hauner H: Diabetesepidemie und Dunkelziffer; in diabetesDE: Deutscher Gesundheitsbericht Diabetes 2010. Mainz, Kirchheim und Co, 2009, pp 8-13.

2 Yan SF, et al: Glycation, inflammation, and RAGE: a scaffold for the macrovascular complications of diabetes and beyond. Circ Res 2003;93:1159-1169.

3 Stettler C, et al: Was Sie schon lange über das $\mathrm{HbA1c}$ wissen wollten. Schweiz Med Wochenschr 2000;130:993-1005.

4 Saraswat M, et al: Prevention of non-enzymic glycation of proteins by dietary agents: prospects for alleviating diabetic complications. $\mathrm{Br} \mathrm{J}$ Nutr 2009;101:1714-1721.

5 Ueberall F, et al: Das anti-inflammatorische Potential von Padma 28 - Übersicht experimenteller Daten zur antiatherogenen Wirkung und Diskussion des Vielstoffkonzepts. Forsch Kom-

plementmed 2006;13(suppl 1):7-12.
Melzer J, et al: Treating intermittent claudication with Tibetan medicine Padma 28: does it work? Atherosclerosis 2006;189:39-46.

Tab. 1. Symptome, Symptomausprägung und Dosierung von PADMA 28 über den Beobachtungszeitraum (Symptomintensität: 3 = stark; 2 = mittelstark; 1 = schwach; $0=$ beschwerdefrei)

\begin{tabular}{lllll}
\hline & Zeitpunkt 0 & 6 Wochen & 5 Monate & 13 Monate \\
\hline Patient 1 (Diabetes mellitus und PAVK) & & & \\
Gefühlsstörungen Füsse & 2 & 1 & 0 & 0 \\
Schmerzen Beine & 2 & 1 & 0 & 0 \\
Dosierung PADMA 28 & $3 \times 2$ & $3 \times 2$ & $3 \times 1$ & $3 \times 1 \mathrm{ff}$. \\
\cline { 2 - 4 } & Zeitpunkt 0 & 6 Wochen & 3 Monate \\
Patient 2 (Diabetes mellitus) & 2 & 1 & 0 \\
Parästhesien Füsse & 2 & 1 & 0 \\
Dysästhesien Hände & $3 \times 2$ & $2 \times 1$ & $2 \times 1 \mathrm{ff}$. \\
Dosierung PADMA 28 & & & \\
\hline
\end{tabular}

